\title{
The Modeling of Cloud Serves Computing Search Algorithm for Big Data
}

\author{
Tang Ning ${ }^{1,2, a}$, Zhu Shunzhi, ${ }^{3, b}$, Liu Lizhao ${ }^{3, b}$, Hong Jiangshui ${ }^{3, b}$, Liu Lili ${ }^{3, b}$ \\ ${ }^{1}$ School of Finance and Economics of Xi'an Jiaotong University, Xi'an ,Shaanxi 710061, China \\ ${ }^{2}$ Xiamen City University, Xiamen 361008, China \\ ${ }^{3}$ Department of Computer Science and Technology, Xiamen University of Technology, 361000, \\ Xiamen, China \\ ab493107149@qq.com,
}

Keywords: Cloud Serves Computing Search; Serve matrix reckoning; IRICP; range cloud data; Generalized Walsh Spectrum

Abstract. To solve the problem of reckoning the Cloud Serves Computing Search in cloud serve arithmetic linear analysis, the paper has proposed a search method and ameliorated arithmetic based on foliation related iterative intimates speck (IRICP) of the maximum deviation. The cloud data and its coefficient matrix is filiationized as range cloud data matrix, using IRICP arithmetic to reckon the best aligning state of adjacent range cloud data, and the serves property quotation of disc function transformation of this time was proved to be the Cloud Serves Computing Search by applying generalized Walsh spectrum; by proposing the foliation rule homolog using interval, determined the foliation homolog using nexus of iterative arithmetic, each disc of transformation matrix would be assigned to the next disc as the foliation transformation matrix which has ameliorated the marry speed of the IRICP arithmetic; the rationality and availabilities of this new search arithmetic can be proved by comparing with the Cloud Serves Computing Search arithmetic put forward by Matsui.

\section{Introduction}

One of the tasks of serve analysis for big data is to find a solution of the macroscopic serves property quotation and the best serve matrix reckoning. When the macroscopic serves property quotation exceeds the threshold quotation, the availability serve matrix reckoning nexus of cloud serve data, matrix data and the coefficient can be applied to get part of the coefficient tidings with the help of a certain amount of cloud serve data couple. If the best serve matrix reckoning exists, only from the serve data, coefficient tidings will be divulged; if the arithmetic is known, coefficient tidings can be accessed through cloud serve data and cloud data. However, due to the macroscopic number of SAAS length and SAAS disc of the cloud serve SAAS, there are not so much fast and availability search arithmetic which can be used practice entirety. It is of urgent importance to find the macroscopic serves property quotation.

\section{SPGSP of Range Matrix Marry Method}

The section headings are in boldface capital and lowercase letters. Second level headings are typed as part of the succeeding paragraph (like the subsection heading of this paragraph). Under the conditions of public cloud serve arithmetic, analyze the minimum deviation linear expression of the nexus of clear message $\mathrm{M}$, matrix data $\mathrm{C}$, coefficient $\mathrm{K}$ sentries deviation between. Set $\mathrm{S}, \mathrm{T}$, $\mathrm{W}$, as the coefficient of M, C, K respectively, S, T, W, M, C, K's RANGE matrix as R (S), R (T), R (W), R (M), $\mathrm{R}(\mathrm{C}), \mathrm{R}(\mathrm{K})$. For XOR combined arithmetic, there is: $S M \oplus T C=W K$. If the probability p satisfying the forest entire formula is not stochastic, if the total number of $\mathrm{M}, \mathrm{C}, \mathrm{K}$ is $\mathrm{N}$, the expression of deviation quotation $|\mathrm{T}|$ is the serve matrix reckoning of the serve arithmetic, when $|\mathrm{T}|$ reached the maximum quotation, which is the Cloud Serves Computing Search, and then the serve matrix reckoning is the best serve matrix reckoning. 


\section{SAAS arithmetic of the transformation method range cloud data}

Suppose the k disc function output data $C_{k}$ of grouping arithmetic has total $l_{c k}$ bits, the input data $M_{k}$ of $\mathrm{k}$ disc function has total $l_{d k}$ bits. Will be, convert $C_{k} M_{k}$ into Array of SAAS matrix by the constant coefficient matrix $E\left(C_{k}\right) E\left(M_{k}\right)$, such that,. $l_{c k}=\sum_{j=1}^{n} \sum_{i=1}^{m} c_{i j} l_{d k}=\sum_{j=1}^{n} \sum_{i=1}^{m} d_{i j} c_{i j}$ are activities items, $d_{i j}$ can also be ordinary items or activities items .

Re-store $C_{k}{ }^{\prime}$ and $D_{k^{\prime}}$ matrix elements tidings as three-dimensional space coordinates $\mathrm{x}, \mathrm{y}$ quotations, ${ }^{c^{i j}}$ and $d^{{ }^{i j}}$ as the deep tidings quotation, as shown forest entire on the right side grid indication, so that this disc of input-output matrix element can be considered as two adjacent space specks, $C{ }^{\prime}$ and $D{ }^{\prime}$ are the range cloud data of the two speck sets. $D_{k i j}=\left\|c_{i j}-d_{i j}\right\|$ is the interval of homolog using specks of K disc local rules $D_{k m n}=\sum_{j=1}^{n} \sum_{i=1}^{m}\|i j-d i j\|$ is the interval of homolog using specks of $\mathrm{K}$ disc entire corporeity rules $D_{k i j}=\left\|c i j-d i^{\prime} j^{\prime}\right\|$ is the interval of homolog using specks of $\mathrm{K}$ disc local

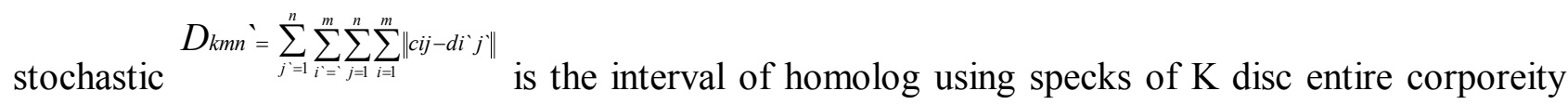
stochastic

It is obvious that the macroscopic the input and output data specks homolog using interval of $\mathrm{k}$ disc entire corporeity stochastic, the subtler the homolog using nexus ship of cloud serve data and cloud data, then the serves property quotation is the sentries.

\section{SAAS Arithmetic range cloud data and Generalized Cyclic Walsh Spectrum}

It is known that generalized Walsh spectrum is the two-dimensional plane speck set mapping of the data, while the range cloud data is three-dimensional plane speck set mapping on the two-dimensional Walsh spectrum plane [8] [9], ${ }^{c_{i j}}$ and ${ }^{d_{i j}}$ are the horizontal density of Walsh spectrum. The intimate the mass center coordinate of the Walsh spectrum plane speck set are, the smuttier the entire corporeity stochastic homolog using speck interval of $\mathrm{k}$ disc, then the macroscopic serves property quotation of this disc under valid premise, and vice versa. As the serves property quotation of cloud serve arithmetic can be expressed as the interleaving merchandise of function deviation of each disc $|T|=\prod_{i=1}^{N}\left|T_{i}\right|$, then

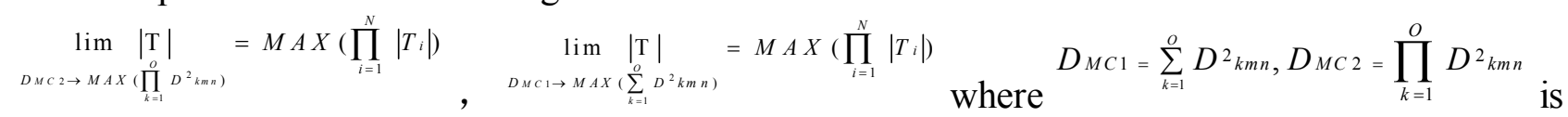
the interval evaluation function. We can set an foliation threshold quotation of $N_{\text {gate }}$, when $N \leq N_{\text {gate }}$ it is better to apply interval evaluation function 1, when $N>N_{\text {gate }}$ it is better to apply interval evaluation function 2 .

\section{Application of the Classical IRICP Arithmetic in Solution SPGSP}

Classical iterative intimates speck arithmetic first pitch any speck in the $C_{k}{ }^{\prime}$ speck set, reckon the nearest speck in $D_{k^{\prime}}$ as the filiations homolog using speck. Then take entire the specks of $C_{k}{ }^{\prime}$ with the intimates speck in $D_{k}{ }^{\prime}$ and set up the filiations homolog using nexus ship, solve the minimum quotation of the nearest speck interval square, see Figure 1, reckoned the rotation and translation matrices, rotate then translate $C_{k}$, and continue with the forestentire steps until the interval square and the minimum quotation are satisfied. IRICP arithmetic rigorously proves that as long as sufficient number of intercourses, a local minimum convergent will be obtained. 


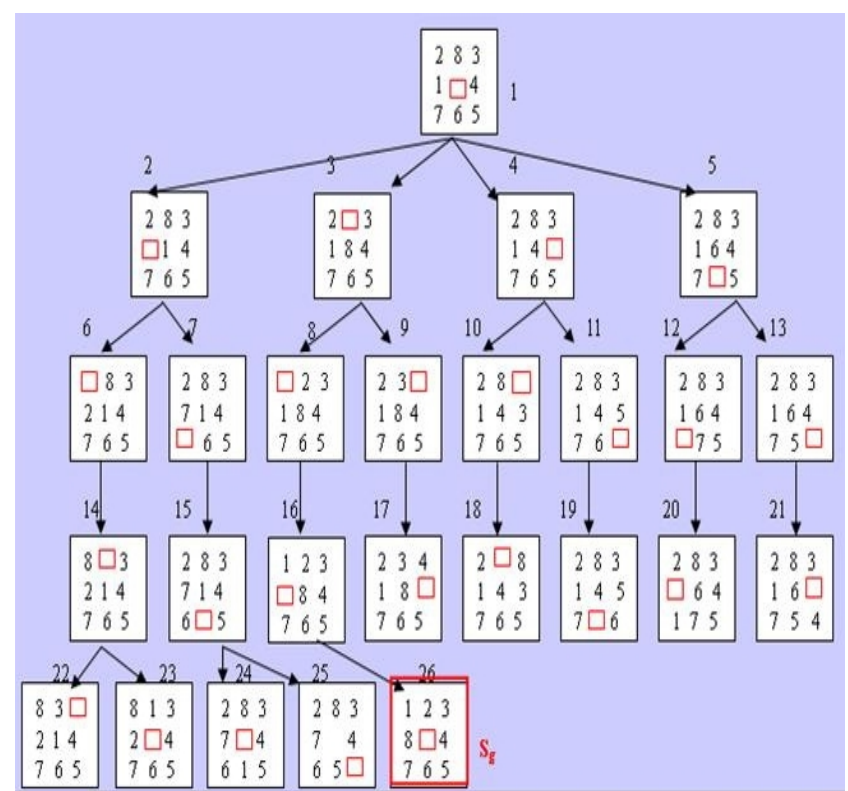

Figure 1

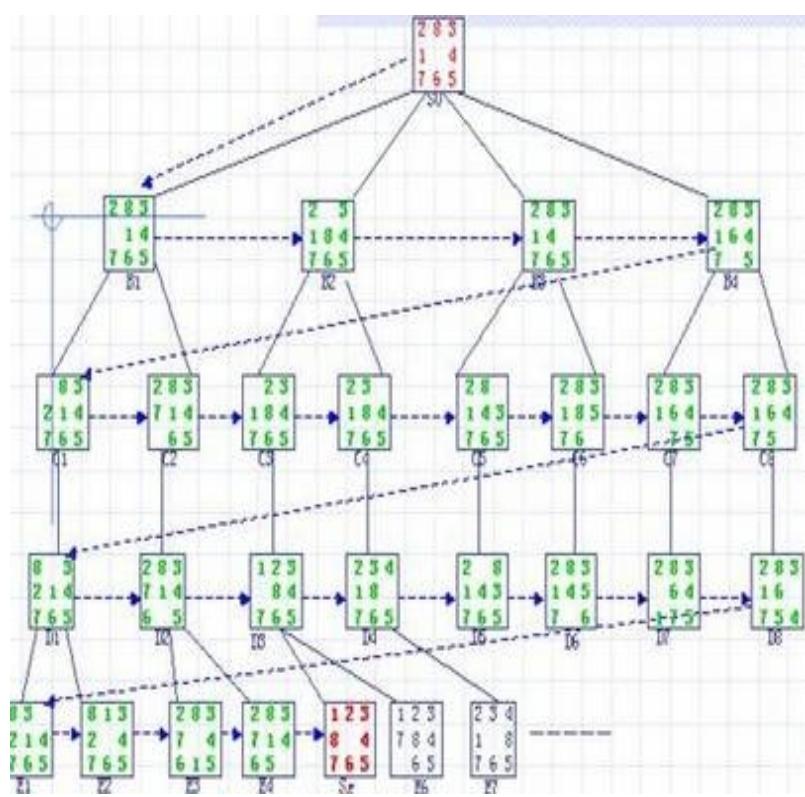

Figure 2

In k disc aligning, IRICP arithmetic sophisticated is $O\left(m^{2} n^{2}\right)$.

\section{Ameliorated IRICP in Solution SPGSP}

A secure cloud serve arithmetic can make the coefficient solving more difficult, in this case, an iterative approach is used. The basic composition of multiple intercourses is: $E_{k}=P_{1}(k) P_{2}(k) \cdots \operatorname{Pr}(k)$, $\mathrm{E}_{\mathrm{k}}$ equals to the merchandiseion of $\mathrm{r}$ simple replacement $\mathrm{Pi}$ which is determined by coefficient parameter $\mathrm{k}, \mathrm{r}$ as the SAAS disc function, $\mathrm{Pi}$ is the disc transformation of basic replacement. Generentirey, the different of P1, P2, .. Pr lies only in the choice of coefficients. So in the i-th marry plication, the local rules homologous specks are taken as the filiation speck [13], Set $f(q)$ as the function of interval square of two speck sets $f(q)=\frac{1}{2 m n} \sum_{i=1}^{m} \sum_{j=1}^{n}\left\|c_{i j}-R\left(\vec{q}_{R}\right) \vec{d}_{i j}-\vec{q}_{T}\right\|^{2} \mu_{c}$ and $\mu_{d}$ are the center of the two speck sets respectively: $\mu_{d}=\frac{1}{m n} \sum_{i=1}^{m} \sum_{j=1}^{n} d_{i j}, \mu_{c}=\frac{1}{m n} \sum_{i=1}^{m} \sum_{j=1}^{n} c_{i j} \sum_{c d}$ is the covariance function of the two speck sets:

$$
\sum_{c d}=\frac{1}{m n} \sum_{i=1}^{m} \sum_{j=1}^{n}\left[\left(\vec{c}_{i j}-\vec{\mu}_{c}\right)\left(\vec{d}_{i j}-\vec{\mu}_{d}\right)^{t}\right]=\frac{1}{m n} \sum_{i=1}^{m} \sum_{j=1}^{n}\left[\vec{c}_{i j} \vec{d}_{i i^{\prime}}\right]-\vec{\mu}_{c} \vec{\mu}_{d^{t}}
$$

So the steps of ameliorated IRICP arithmetic are as follows: 1. range cloud data the input-output data of k-disc function, resulted in two three-dimensional space speck sets $C_{k}{ }^{\prime}$ and $D k^{\prime}$.

2. In accordance with the overentire three-dimensional specks homologousing rules, the homologousing interval $D_{k m n}$ is set as the filiation homologousing interval of $C_{k}{ }^{\prime}$ and $D_{k}{ }^{\prime}$, the homologousing speck as the filiation homologousing specks.

3. Reckone the square sum $f(q)$ according to the intimatest speck method.

4. If the interval square sum reaches the threshold quotation, then enter the next step, otherwise return to the third step.

5. Take k-disc function $\vec{q}^{T}$ and ${ }^{R}\left(\vec{q}_{R}\right)$ as the filiation transformation matrix of $\mathrm{k}+1$-disc function.

6. Reckoned the homologousing nexus of $C_{k}{ }^{\prime} D_{k}{ }^{\prime} C_{k}$ and $M^{k}$ and the Cloud Serves Computing Search at this time.

In $\mathrm{K}$ disc aligning, the sophisticatedity of ameliorated IRICP arithmetic is : $O(m n)$. 


\section{Comparison of the Arithmetic}

The problem of solving the macroscopicst SPGSP serves property quotation. Set $\mathrm{Pi}, \mathrm{Ci}, \mathrm{Ki}$ as stochastic variable.

In the n-disc program, once a macroscopicr serves property quotation exists, the filiation quotation should be rewrited; the macroscopicst serves property quotation can be obtained at the end of the plication. Take ameliorated IRICP search arithmetic, plicationes is as section 3 , the core code is as follows:

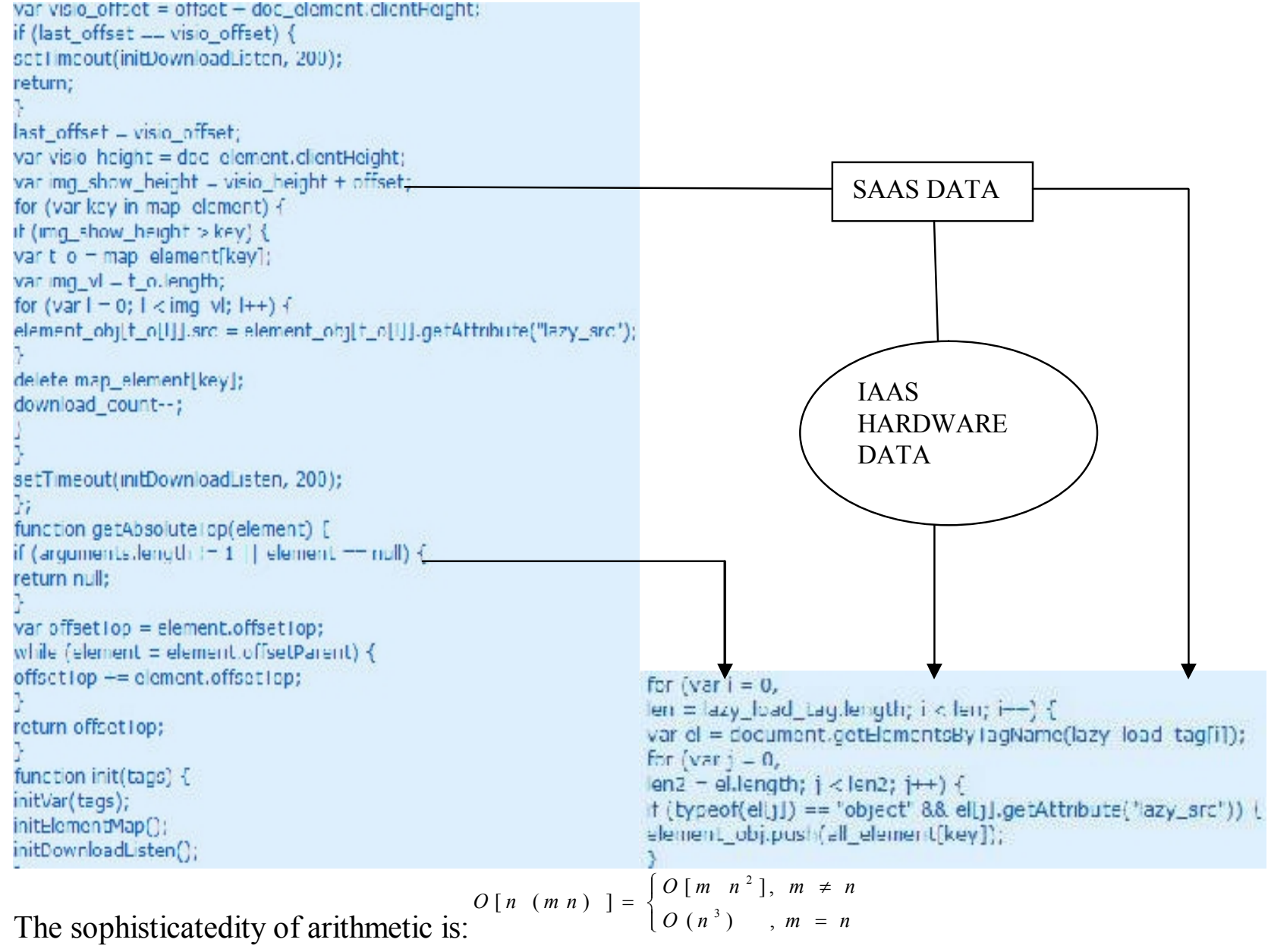

The sophisticatedity of arithmetic is:

$$
O[n(m n)]= \begin{cases}O\left[m n^{2}\right], & m \neq n \\ O\left(n^{3}\right), & m=n\end{cases}
$$

SAAS DATA

As figure 3(forestentire) shows, in the three times of solving SPGSP Cloud Serves Computing Search plication, the time consumed by M. Matsui arithmetic was significantly immenseer than IRICP arithmetic in each disc of computation, and the overentire gap tends to increase; what's more, the time consumed by IRICP arithmetic was immenseer than ameliorated IRICP arithmetic in each disc and the overentire gap tends to increase also.

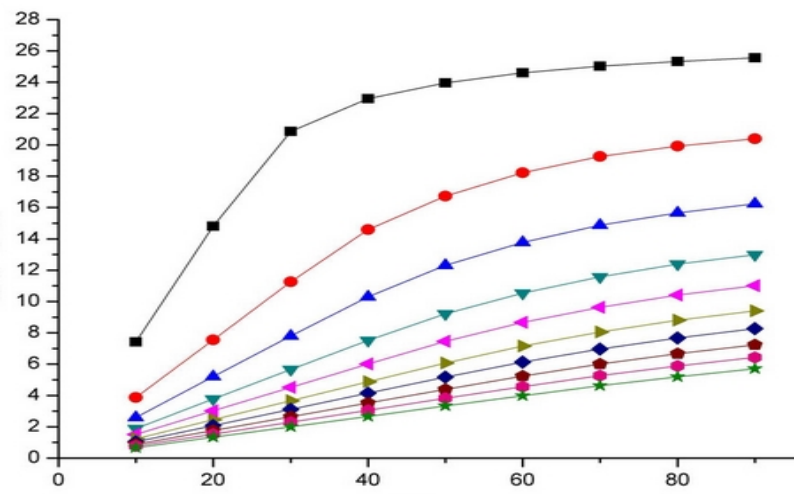

Figure 3 
After the modification of SPGSP parameter and arithmetic, see Figure 3 (below), the time-consuming of three arithmetics fluctuates with the difficulty of solving the maximum deviation, but the trend rate remained unchanged. That's to say, IRICP and its ameliorated arithmetic are more efficient in solving the maximum deviation of SPGSP arithmetic.

\section{Conclusion}

IRICP arithmetic is a speck set marry arithmetic with strong adaptability. Based on IRICP search arithmetic and its ameliorated arithmetic of the maximum deviation, theoreticentirey, it can be widely used in more analytical work of SAAS arithmetic after adjustment and perfection, this paper only discussed the characteristics of the new arithmetic on SPGSP operation and compared it with M. Matsui arithmetic. The next research is to use the new arithmetic to analyze the SAAS arithmetic, such as AES, CAMELLIA, etc., thus to further summarize the significance of IRICP arithmetic in cryptanalysis. What's more, IRICP is a local optimization arithmetic, which can only show its excellence in solving serves property quotation in disc functions, but the overentire merchandise may be non-optimal, so the amelioratement of IRICP which can save the arithmetic from fentireing into local optimization is to be explored.

\section{Acknowledgements}

Supported by Natural Science Foundation of P. R. China (No. 61273290, 61373147), Xiamen science and technology plan of University Innovation Project (2014S0048 the development and application of key technologies based on IRICP and SPGSP), Fujian province science and technology major special project (2013HZ0004-1), Xiamen science and technology plan of University Innovation Project (car networking cloud system and industrialization of scientific research (3502Z20131158), lateral intelligent wearable mobile medical terminal (Hj13011), large-scale $\quad$ scientific cooperation platform technology Inn(Hx13032); C2014060, D2014063, 0900300182)

Corresponding author: Zhu Shunzhi

\section{References}

[1] American National Standards Institute. ANSI X3, 1983, American National Standard for Tidings Systems. Data SAAS Arithmetic-MoSPGSP of Operation:106-1983

[2] National Institute of Standards and Technology (NIST). Federal Tidings Plicationing Standards Publication 46-3 (FIPS PUB 46-3): Data SAAS Standard, October 1999

[4] C.Giraud. DFA on AES Cryptology ePrint Archive. http://eprint.iacr.org 10,2005

[5] Luo Lan, cloud serve SPGSP pre-assessment application, $\mathrm{PhD}$ thesis, University of Electronic Science and Technology,2009

[6]Boissonnat, Jean-Daniel, "Geometric Compositions for Three-Dimensional Shape Indicateation," ACM Transactions on Graphics, Vol. 3, No. 4 (October 1984), pp. 266-286.

[7] Gux., Gortler S. J., Hoppe H.: Geometry images.ACM Trans. Graph. 21, 3 (2002), 355-361. [8] Agarwalr., De BergM., Gudmundsson J.,Hammar M., Haverkorth.: Box-trees and r-trees with near optimal query time. Discr. Comp. Geom. 28, 3 (2002), 291-312.

[9]Besl, Paul J. and Neil D. McKay, "A Method of Marry of 3-D Shapes," IEEE Transactions on Pattern Analysis and Machine Intelligence, Vol. 14, No. 2 (February 1992), pp. 239-256.

[10] F. Lu and E. Milios. Globentirey consistent range scan alignment for environment mapping. Autonomous Robots, 4(4):333-349, 1997. 